\title{
An Improvement of Ab Initio Ligand Field Theory by Means of Multistate Perturbation Theory
}

\section{Supporting Information}

Lucas Lang, ${ }^{\mathrm{a}}$ Mihail Atanasov, ${ }^{\mathrm{a}, \mathrm{b}}$ and Frank Neese*,a

${ }^{a}$ Max-Planck-Institut für Kohlenforschung, Kaiser-Wilhelm-Platz 1, 45470 Mülheim an

der Ruhr, Germany

${ }^{b}$ Institute of General and Inorganic Chemistry, Bulgarian Academy of Sciences, Akad.

Georgi Bontchev Street 11, 1113 Sofia, Bulgaria 


\section{Results for the $\mathrm{Cr}^{\mathrm{III}}$ series with only quartet roots included}

Table S1: Quartet excitation energies (in eV) for the $\mathrm{Cr} X_{6}$ series obtained with different geometries and averaged over only the quartet roots.

\begin{tabular}{cccccc}
\hline \hline & \multicolumn{2}{c}{ gas phase structure } & \multicolumn{2}{c}{ C-PCM structure } & \\
& CASSCF & NEVPT2 & CASSCF & NEVPT2 & Expt. \\
\hline$\left[\mathrm{CrF}_{6}\right]^{3-}$ & 1.41 & 1.62 & 1.64 & 1.86 & $2.00^{\mathrm{a}}$ \\
& 2.32 & 2.56 & 2.64 & 2.86 & $2.90^{\mathrm{a}}$ \\
& 3.95 & 3.92 & 4.31 & 4.32 & $\ldots$ \\
\hline$\left[\mathrm{CrCl}_{6}\right]^{3-}$ & 1.18 & 1.48 & 1.36 & 1.69 & $1.70^{\mathrm{b}}$ \\
& 1.97 & 2.37 & 2.23 & 2.62 & $2.38^{\mathrm{b}}$ \\
& 3.53 & 3.58 & 3.79 & 3.91 & $\ldots$ \\
\hline$\left[\mathrm{CrBr}_{6}\right]^{3-}$ & 1.10 & 1.46 & 1.25 & 1.64 & $1.66^{\mathrm{c}}$ \\
& 1.85 & 2.34 & 2.07 & 2.55 & $2.16^{\mathrm{c}}$ \\
& 3.40 & 3.51 & 3.61 & 3.79 & $\ldots$ \\
\hline$\left[\mathrm{CrI}_{6}\right]^{3-}$ & 1.09 & 1.57 & 1.22 & 1.73 & $\ldots$ \\
& 1.84 & 2.47 & 2.03 & 2.65 & $\ldots$ \\
\hline$\left[\mathrm{Cr}\left(\mathrm{CN}_{6}\right)^{3-}\right.$ & 3.36 & 3.62 & 3.55 & 3.88 & $\ldots$ \\
\hline $\left.\mathrm{Cr}^{3-}\left(\mathrm{NH}_{3}\right)_{6}\right]^{3+}$ & 3.00 & 3.61 & 3.37 & 4.04 & $3.29^{\mathrm{d}}$ \\
& 4.20 & 4.64 & 4.57 & 5.05 & $4.02^{\mathrm{d}}$
\end{tabular}

a: $\mathrm{K}_{2} \mathrm{NaCrF}_{6}{ }^{1}$ This system has an experimental metal-ligand distance that is closest to the CPCM geometry among the systems in Table 1 of the main manuscript.

b: $\mathrm{CrCl}_{3}{ }^{1}$.

c: $\mathrm{CrBr}_{3}{ }^{1}$.

$\mathrm{d}: \mathrm{K}{ }_{3} \mathrm{Cr}(\mathrm{CN})_{6}{ }^{2}$.

e: $\left[\mathrm{Cr}\left(\mathrm{NH}_{3}\right)_{6}\right]\left(\mathrm{ClO}_{4}\right)_{3}$ in $\mathrm{H}_{2} \mathrm{O}^{3,4}$. 
2 Representatives of orbitally degenerate quartet states of octahedral $\mathrm{d}^{3}$ complexes

It is possible to investigate an octahedral system in the $\mathrm{D}_{4 \mathrm{~h}}$ subgroup. In this case $\mathrm{xz}, \mathrm{yz}$ belong to the $E_{g}$, xy to the $B_{2 g}$, $z^{2}$ to the $A_{1 g}$, and $x^{2}-y^{2}$ to the $B_{1 g}$ irreducible representations. A state that has a half-filled $\mathrm{E}_{\mathrm{g}}$ shell, i.e. both the $\mathrm{xz}$ and the $\mathrm{yz}$ orbital singly occupied with a spin-up electron, has symmetry $A_{2 g}$. The ground state, with $x y, x z$ and yz singly occupied, therefore has $A_{2 g} \otimes B_{2 g}=B_{1 g}$ symmetry. The singly excited Slater determinants have the following symmetries:

- $\quad \mathrm{xy} \rightarrow \mathrm{z}^{2}: A_{2 g} \otimes A_{1 g}=A_{2 g}$

- $\quad \mathrm{xy} \rightarrow \mathrm{x}^{2}-\mathrm{y}^{2}: A_{2 g} \otimes B_{1 g}=B_{2 g}$

- $\mathrm{xz} / \mathrm{yz} \rightarrow \mathrm{z}^{2}: E_{g} \otimes B_{2 g} \otimes A_{1 g}=E_{g}$

- $\mathrm{xz} / \mathrm{yz} \rightarrow \mathrm{x}^{2}-\mathrm{y}^{2}: E_{g} \otimes B_{2 g} \otimes B_{1 g}=E_{g}$

The doubly excited Slater determinants have the following symmetries:

- $\quad \mathrm{xz}, \mathrm{yz} \rightarrow \mathrm{z}^{2}, \mathrm{x}^{2}-\mathrm{y}^{2}: B_{2 g} \otimes A_{1 g} \otimes B_{1 g}=A_{2 g}$ 
- $\quad \mathrm{xz} / \mathrm{yz}, \mathrm{xy} \rightarrow \mathrm{z}^{2}, \mathrm{x}^{2}-\mathrm{y}^{2}: E_{g} \otimes A_{1 g} \otimes B_{1 g}=E_{g}$

The irreducible representations of $\mathrm{O}_{\mathrm{h}}$ transform in the $\mathrm{D}_{4 \mathrm{~h}}$ subgroup as

- $\mathrm{A}_{2 \mathrm{~g}} \rightarrow \mathrm{B}_{1 \mathrm{~g}}$

- $\mathrm{T}_{1 \mathrm{~g}} \rightarrow A_{2 g} \oplus E_{g}$

- $\mathrm{T}_{2 \mathrm{~g}} \rightarrow B_{2 g} \oplus E_{g}$

One can see that this is in accordance with the above results and that the $x y \rightarrow x^{2}-y^{2}$

excited Slater determinant is one component of the ${ }^{4} \mathrm{~T}_{2 \mathrm{~g}}$ excited level, while the $\mathrm{xy} \rightarrow \mathrm{z}^{2}$ and $\mathrm{xz}, \mathrm{yz} \rightarrow \mathrm{z}^{2}, \mathrm{x}^{2}-\mathrm{y}^{2}$ excited Slater Determinants will mix to form one component of each of the two excited ${ }^{4} \mathrm{~T}_{1 \mathrm{~g}}$ levels.

\section{Additional $a b$ initio effective Hamiltonians}

The quartet block of the DCD-CAS(2) effective Hamiltonian in the CSF basis is

$$
\mathbf{H}_{\mathrm{DCD}-\mathrm{CAS}(2)}^{\mathrm{eff}}=\left(\begin{array}{cccc}
0 & 0 & 0 & 0 \\
0 & 1.880 & 0 & 0 \\
0 & 0 & 3.168 & 0.652 \\
0 & 0 & 0.652 & 4.052
\end{array}\right)
$$

for the $\left[\mathrm{CrF}_{6}\right]^{3-}$ complex, 


$$
\mathbf{H}_{\mathrm{DCD}-\mathrm{CAS}(2)}^{\text {eff }}=\left(\begin{array}{cccc}
0 & 0 & 0 & 0 \\
0 & 1.682 & 0 & 0 \\
0 & 0 & 2.844 & 0.603 \\
0 & 0 & 0.603 & 3.640
\end{array}\right)
$$

for the $\left[\mathrm{CrCl}_{6}\right]^{3-}$ complex and

$$
\mathbf{H}_{\mathrm{DCD}-\mathrm{CAS}(2)}^{\text {eff }}=\left(\begin{array}{cccc}
0 & 0 & 0 & 0 \\
0 & 1.638 & 0 & 0 \\
0 & 0 & 2.767 & 0.591 \\
0 & 0 & 0.591 & 3.550
\end{array}\right)
$$

for the $\left[\mathrm{CrBr}_{6}\right]^{3-}$ complex.

4 Exact numerical data for RMSDs and trends in ligand field parameters

Table S2: Total RMSDs (in eV) between AILFT and ab initio state energies.

\begin{tabular}{lcccc}
\hline \hline & CASSCF & NEVPT2 & HQD-NEVPT2 & DCD-CAS(2) \\
\hline$\left[\mathrm{CrF}_{6}\right]^{3-}$ & 0.01 & 0.25 & 0.09 & 0.08 \\
{$\left[\mathrm{CrCl}_{6}\right]^{3-}$} & 0.10 & 0.41 & 0.20 & 0.18 \\
{$\left[\mathrm{CrBr}_{6}\right]^{3-}$} & 0.12 & 0.48 & 0.25 & 0.23 \\
{$\left[\mathrm{CrI}_{6}\right]^{3-}$} & 0.15 & 0.61 & 0.33 & 0.31 \\
{$\left[\mathrm{Cr}(\mathrm{CN})_{6}\right]^{3-}$} & 0.08 & 0.24 & 0.17 & 0.11 \\
{$\left[\mathrm{Cr}\left(\mathrm{NH}_{3}\right)_{6}\right]^{3+}$} & 0.08 & 0.32 & 0.17 & 0.13 \\
{$\left[\mathrm{MoCl}_{6}\right]^{-}$} & 0.10 & 0.19 & 0.13 & 0.10 \\
{$\left[\mathrm{TcF}_{6}\right]^{--}$} & 0.07 & 0.16 & 0.14 & 0.09 \\
$\mathrm{IrF}_{6}$ & 0.12 & 0.25 & 0.26 & 0.07 \\
& & $\mathrm{~S} 5$ & &
\end{tabular}




\begin{tabular}{lllll}
{$\left[\mathrm{ReF}_{6}\right]^{2-}$} & 0.05 & 0.15 & 0.16 & 0.10 \\
{$\left[\mathrm{ReCl}_{6}\right]^{2-}$} & 0.14 & 0.30 & 0.31 & 0.23 \\
{$\left[\mathrm{ReBr}_{6}\right]^{2-}$} & 0.15 & 0.40 & 0.41 & 0.31 \\
{$\left[\mathrm{TiCl}_{4}\right]^{2-}$} & 0.05 & 0.09 & 0.08 & 0.07 \\
{$\left[\mathrm{VCl}_{4}\right]^{2-}$} & 0.03 & 0.14 & 0.10 & 0.08 \\
{$\left[\mathrm{CrCl}_{4}\right]^{2-}$} & 0.02 & 0.31 & 0.16 & 0.14 \\
{$\left[\mathrm{MnCl}_{4}\right]^{2-}$} & 0.01 & 0.17 & 0.19 & 0.16 \\
{$\left[\mathrm{FeCl}_{4}\right]^{2-}$} & 0.02 & 0.16 & 0.13 & 0.12 \\
{$\left[\mathrm{CoCl}_{4}\right]^{2-}$} & 0.02 & 0.17 & 0.07 & 0.06 \\
{$\left[\mathrm{NiCl}_{4}\right]^{2-}$} & 0.03 & 0.13 & 0.08 & 0.07 \\
$\left.\mathrm{Cr}^{2 \mathrm{acac}}\right)_{3}$ & 0.01 & 0.29 & 0.11 & 0.09 \\
$\mathrm{Mn}_{3}(\mathrm{acac})_{3}$ & 0.05 & 0.41 & 0.23 & 0.20 \\
{$\left[\mathrm{FeO}_{4}\right]^{2-}$} & 0.32 & 1.32 & 1.37 & 1.16 \\
{$\left[\mathrm{MnO}_{4}\right]^{3-}$} & 0.14 & 0.53 & 0.51 & 0.45 \\
\hline \hline
\end{tabular}

Table S3: Ligand field splittings $\Delta$ (in eV) for all complexes in the test set that are approximately octahedral or tetrahedral. All ligand field orbital energies belonging to degenerate sets in the pseudo symmetry group were averaged.

\begin{tabular}{lcccc}
\hline \hline & CASSCF & NEVPT2 & HQD-NEVPT2 & DCD-CAS(2) \\
\hline$\left[\mathrm{CrF}_{6}\right]^{3-}$ & 1.64 & 1.65 & 1.84 & 1.84 \\
{$\left[\mathrm{CrCl}_{6}\right]^{3-}$} & 1.30 & 1.39 & 1.61 & 1.56 \\
{$\left[\mathrm{CrBr}_{6}\right]^{3-}$} & 1.18 & 1.30 & 1.54 & 1.49 \\
{$\left[\mathrm{CrI}_{6}\right]^{3-}$} & 1.13 & 1.31 & 1.60 & 1.51 \\
{$\left[\mathrm{Cr}(\mathrm{CN})_{6}\right]^{3-}$} & 3.32 & 3.84 & 4.00 & 3.97 \\
$\left.\left[\mathrm{Cr}^{3-} \mathrm{NH}_{3}\right)_{6}\right]^{3+}$ & 2.36 & 2.54 & 2.78 & 2.75 \\
{$\left[\mathrm{MoCl}_{6}\right]^{3-}$} & 2.15 & 2.22 & 2.33 & 2.34 \\
{$\left[\mathrm{TcF}_{6}\right]^{2-}$} & 3.46 & 3.56 & 3.63 & 3.69 \\
$\mathrm{IrF}_{6}$ & 5.33 & 4.92 & 4.94 & 5.24 \\
{$\left[\mathrm{ReF}_{6}\right]^{2-}$} & 3.98 & 4.01 & 4.05 & 4.13 \\
{$\left[\mathrm{ReCl}_{6}\right]^{2-}$} & 3.16 & 3.27 & 3.32 & 3.35 \\
{$\left[\mathrm{ReBr}_{6}\right]^{2-}$} & 2.86 & 3.00 & 3.06 & 3.09 \\
{$\left[\mathrm{TiCl}_{4}\right]^{2-}$} & 0.40 & 0.38 & 0.40 & 0.39
\end{tabular}




\begin{tabular}{lllll}
{$\left[\mathrm{MnCl}_{4}\right]^{2-}$} & 0.33 & 0.29 & 0.38 & 0.38 \\
{$\left[\mathrm{FeCl}_{4}\right]^{2-}$} & 0.33 & 0.32 & 0.42 & 0.42 \\
{$\left[\mathrm{CoCl}_{4}\right]^{2-}$} & 0.33 & 0.33 & 0.44 & 0.44 \\
$\mathrm{Cr}(\mathrm{acac})_{3}$ & 2.05 & 2.11 & 2.35 & 2.34 \\
{$\left[\mathrm{FeO}_{4}\right]^{2-}$} & 2.11 & 2.13 & 2.31 & 1.70 \\
{$\left[\mathrm{MnO}_{4}\right]^{3-}$} & 1.69 & 1.83 & 2.01 & 1.81 \\
\hline
\end{tabular}

Table S4: AlLFT Racah parameter B (in eV) derived from different ab initio methods for all complexes in the test set.

\begin{tabular}{|c|c|c|c|c|}
\hline & CASSCF & NEVPT2 & HQD-NEVPT2 & DCD-CAS(2) \\
\hline$\left[\mathrm{CrF}_{6}\right]^{3-}$ & 0.133 & 0.121 & 0.109 & 0.111 \\
\hline$\left[\mathrm{CrCl}_{6}\right]^{3-}$ & 0.124 & 0.110 & 0.099 & 0.100 \\
\hline$\left[\mathrm{CrBr}_{6}\right]^{3-}$ & 0.121 & 0.106 & 0.095 & 0.098 \\
\hline$\left[\mathrm{CrI}_{6}\right]^{3-}$ & 0.117 & 0.102 & 0.090 & 0.093 \\
\hline$\left[\mathrm{Cr}(\mathrm{CN})_{6}\right]^{3-}$ & 0.113 & 0.104 & 0.089 & 0.090 \\
\hline$\left[\mathrm{Cr}\left(\mathrm{NH}_{3}\right)_{6}\right]^{3+}$ & 0.125 & 0.122 & 0.102 & 0.102 \\
\hline$\left[\mathrm{MoCl}_{6}\right]^{3-}$ & 0.081 & 0.068 & 0.056 & 0.057 \\
\hline$\left[\mathrm{TcF}_{6}\right]^{2-}$ & 0.094 & 0.077 & 0.065 & 0.067 \\
\hline $\operatorname{IrF}_{6}$ & 0.085 & 0.051 & 0.045 & 0.046 \\
\hline$\left[\operatorname{ReF}_{6}\right]^{2-}$ & 0.088 & 0.072 & 0.066 & 0.066 \\
\hline$\left[\mathrm{ReCl}_{6}\right]^{2-}$ & 0.072 & 0.060 & 0.052 & 0.053 \\
\hline$\left[\operatorname{ReBr}_{6}\right]^{2-}$ & 0.067 & 0.057 & 0.049 & 0.050 \\
\hline$\left[\mathrm{TiCl}_{4}\right]^{2-}$ & 0.095 & 0.080 & 0.078 & 0.078 \\
\hline$\left[\mathrm{VCl}_{4}\right]^{2-}$ & 0.107 & 0.088 & 0.085 & 0.086 \\
\hline$\left[\mathrm{CrCl}_{4}\right]^{2-}$ & 0.118 & 0.104 & 0.095 & 0.097 \\
\hline$\left[\mathrm{MnCl}_{4}\right]^{2-}$ & 0.130 & 0.105 & 0.105 & 0.106 \\
\hline$\left[\mathrm{FeCl}_{4}\right]^{2-}$ & 0.138 & 0.113 & 0.113 & 0.114 \\
\hline$\left[\mathrm{CoCl}_{4}\right]^{2-}$ & 0.146 & 0.122 & 0.122 & 0.123 \\
\hline$\left[\mathrm{NiCl}_{4}\right]^{2-}$ & 0.154 & 0.137 & 0.135 & 0.134 \\
\hline $\mathrm{Cr}(\mathrm{acac})_{3}$ & 0.128 & 0.123 & 0.103 & 0.105 \\
\hline $\operatorname{Mn}(\operatorname{acac})_{3}$ & 0.136 & 0.124 & 0.110 & 0.113 \\
\hline
\end{tabular}




\begin{tabular}{lllll}
{$\left[\mathrm{FeO}_{4}\right]^{2-}$} & 0.081 & 0.101 & 0.047 & 0.058 \\
{$\left[\mathrm{MnO}_{4}\right]^{3-}$} & 0.098 & 0.105 & 0.064 & 0.071 \\
\hline \hline
\end{tabular}

Table S5: AILFT Racah parameter C (in eV) derived from different ab initio methods for all complexes in the test set.

\begin{tabular}{|c|c|c|c|c|}
\hline & CASSCF & NEVPT2 & HQD-NEVPT2 & $\mathrm{DCD}-\mathrm{CAS}(2)$ \\
\hline$\left[\mathrm{CrF}_{6}\right]^{3-}$ & 0.499 & 0.426 & 0.446 & 0.448 \\
\hline$\left[\mathrm{CrCl}_{6}\right]^{3-}$ & 0.471 & 0.397 & 0.414 & 0.416 \\
\hline$\left[\mathrm{CrBr}_{6}\right]^{3-}$ & 0.467 & 0.391 & 0.405 & 0.407 \\
\hline$\left[\mathrm{CrI}_{6}\right]^{3-}$ & 0.459 & 0.373 & 0.387 & 0.390 \\
\hline$\left[\mathrm{Cr}(\mathrm{CN})_{6}\right]^{3-}$ & 0.435 & 0.363 & 0.361 & 0.371 \\
\hline$\left[\mathrm{Cr}\left(\mathrm{NH}_{3}\right)_{6}\right]^{3+}$ & 0.476 & 0.393 & 0.416 & 0.422 \\
\hline$\left[\mathrm{MoCl}_{6}\right]^{3-}$ & 0.329 & 0.262 & 0.276 & 0.280 \\
\hline$\left[\mathrm{TcF}_{6}\right]^{2-}$ & 0.374 & 0.307 & 0.320 & 0.326 \\
\hline $\mathrm{IrF}_{6}$ & 0.294 & 0.215 & 0.226 & 0.252 \\
\hline$\left[\mathrm{ReF}_{6}\right]^{2-}$ & 0.353 & 0.302 & 0.305 & 0.313 \\
\hline$\left[\mathrm{ReCl}_{6}\right]^{2-}$ & 0.303 & 0.244 & 0.246 & 0.256 \\
\hline$\left[\mathrm{ReBr}_{6}\right]^{2-}$ & 0.297 & 0.225 & 0.227 & 0.238 \\
\hline$\left[\mathrm{TiCl}_{4}\right]^{2-}$ & 0.364 & 0.296 & 0.297 & 0.300 \\
\hline$\left[\mathrm{VCl}_{4}\right]^{2-}$ & 0.407 & 0.346 & 0.346 & 0.349 \\
\hline$\left[\mathrm{CrCl}_{4}\right]^{2-}$ & 0.444 & 0.379 & 0.386 & 0.390 \\
\hline$\left[\mathrm{MnCl}_{4}\right]^{2-}$ & 0.487 & 0.428 & 0.425 & 0.427 \\
\hline$\left[\mathrm{FeCl}_{4}\right]^{2-}$ & 0.512 & 0.459 & 0.452 & 0.456 \\
\hline$\left[\mathrm{CoCl}_{4}\right]^{2-}$ & 0.543 & 0.491 & 0.482 & 0.484 \\
\hline$\left[\mathrm{NiCl}_{4}\right]^{2-}$ & 0.572 & 0.500 & 0.501 & 0.504 \\
\hline $\mathrm{Cr}(\mathrm{acac})_{3}$ & 0.484 & 0.398 & 0.426 & 0.429 \\
\hline $\operatorname{Mn}(\operatorname{acac})_{3}$ & 0.515 & 0.438 & 0.453 & 0.456 \\
\hline$\left[\mathrm{FeO}_{4}\right]^{2-}$ & 0.394 & -0.060 & 0.056 & 0.067 \\
\hline$\left[\mathrm{MnO}_{4}\right]^{3-}$ & 0.402 & 0.183 & 0.269 & 0.271 \\
\hline
\end{tabular}


Table S6: AILFT Racah parameter ratio C/B derived from different ab initio methods for all complexes in the test set.

\begin{tabular}{|c|c|c|c|c|}
\hline & CASSCF & NEVPT2 & HQD-NEVPT2 & DCD-CAS $(2)$ \\
\hline$\left[\mathrm{CrF}_{6}\right]^{3-}$ & 3.75 & 3.51 & 4.07 & 4.05 \\
\hline$\left[\mathrm{CrCl}_{6}\right]^{3-}$ & 3.81 & 3.61 & 4.20 & 4.14 \\
\hline$\left[\mathrm{CrBr}_{6}\right]^{3-}$ & 3.85 & 3.69 & 4.21 & 4.18 \\
\hline$\left[\mathrm{CrI}_{6}\right]^{3-}$ & 3.93 & 3.67 & 4.31 & 4.19 \\
\hline$\left[\mathrm{Cr}(\mathrm{CN})_{6}\right]^{3-}$ & 3.84 & 3.49 & 4.12 & 4.06 \\
\hline$\left[\mathrm{Cr}\left(\mathrm{NH}_{3}\right)_{6}\right]^{3+}$ & 3.82 & 3.22 & 4.09 & 4.14 \\
\hline$\left[\mathrm{MoCl}_{6}\right]^{3-}$ & 4.07 & 3.84 & 4.92 & 4.92 \\
\hline$\left[\mathrm{TcF}_{6}\right]^{2-}$ & 3.99 & 4.01 & 4.89 & 4.89 \\
\hline $\mathrm{IrF}_{6}$ & 3.46 & 4.22 & 5.06 & 5.43 \\
\hline$\left[\mathrm{ReF}_{6}\right]^{2-}$ & 4.02 & 4.17 & 4.64 & 4.71 \\
\hline$\left[\mathrm{ReCl}_{6}\right]^{2-}$ & 4.23 & 4.09 & 4.73 & 4.86 \\
\hline$\left[\mathrm{ReBr}_{6}\right]^{2-}$ & 4.46 & 3.94 & 4.63 & 4.80 \\
\hline$\left[\mathrm{TiCl}_{4}\right]^{2-}$ & 3.85 & 3.71 & 3.79 & 3.86 \\
\hline$\left[\mathrm{VCl}_{4}\right]^{2-}$ & 3.79 & 3.96 & 4.05 & 4.07 \\
\hline$\left[\mathrm{CrCl}_{4}\right]^{2-}$ & 3.75 & 3.65 & 4.05 & 4.03 \\
\hline$\left[\mathrm{MnCl}_{4}\right]^{2-}$ & 3.73 & 4.06 & 4.04 & 4.03 \\
\hline$\left[\mathrm{FeCl}_{4}\right]^{2-}$ & 3.72 & 4.06 & 3.99 & 4.00 \\
\hline$\left[\mathrm{CoCl}_{4}\right]^{2-}$ & 3.71 & 4.03 & 3.94 & 3.95 \\
\hline$\left[\mathrm{NiCl}_{4}\right]^{2-}$ & 3.71 & 3.66 & 3.71 & 3.75 \\
\hline $\mathrm{Cr}(\mathrm{acac})_{3}$ & 3.77 & 3.25 & 4.12 & 4.08 \\
\hline $\operatorname{Mn}(\operatorname{acac})_{3}$ & 3.77 & 3.54 & 4.10 & 4.03 \\
\hline$\left[\mathrm{FeO}_{4}\right]^{2-}$ & 4.87 & -0.59 & 1.21 & 1.16 \\
\hline$\left[\mathrm{MnO}_{4}\right]^{3-}$ & 4.08 & 1.75 & 4.22 & 3.83 \\
\hline
\end{tabular}




\section{Derivation of Eq. 13 of the main manuscript}

An arbitrary real $2 \times 2$ Hermitian matrix can be written

$$
\mathbf{H}=E_{0} \mathbf{I}+\left(\begin{array}{cc}
-\Delta E / 2 & V \\
V & \Delta E / 2
\end{array}\right),
$$

where $E_{0}$ is a constant shift that does not influence the eigenvectors, I is the $2 \times 2$ identity matrix, $\Delta E$ is the difference between the diagonal elements, and $V$ is the offdiagonal coupling matrix element. Diagonalizing $\mathbf{H}$, i.e. solving its eigenvalue equation,

leads to two solutions with energies

$$
E_{ \pm}= \pm \frac{1}{2} \sqrt{(\Delta E)^{2}+4 V^{2}}
$$

The eigenvector belonging to the lower-energy solution $E_{-}$can be written as

$$
\left(\begin{array}{l}
C_{1} \\
C_{2}
\end{array}\right)=N\left(\begin{array}{c}
-\left(\Delta E / V+\sqrt{(\Delta E / V)^{2}+4}\right) / 2 \\
1
\end{array}\right)
$$

where $N$ is an arbitrary normalization constant. This means that

$$
-\left(\Delta E / V+\sqrt{(\Delta E / V)^{2}+4}\right) / 2=C_{1} / C_{2} .
$$

Solving this equation for $\Delta E / V$ gives 


$$
\frac{\Delta E}{V}=\frac{C_{2}}{C_{1}}-\frac{C_{1}}{C_{2}}
$$

For the $2 \times 2{ }^{4} \mathrm{~T}_{1 \mathrm{~g}}$ block of the LFT Hamiltonian of an octahedral $\mathrm{d}^{3}$ complex (see Eq.

(12) of the main manuscript), one has

$$
\frac{\Delta E}{V}=\frac{\Delta-9 B}{6 B}=\frac{\Delta / B-9}{6},
$$

which leads, combined with Eq. (8), to Eq. (13) of the main manuscript,

$$
\frac{\Delta}{B}=6 \frac{\Delta E}{V}+9=6\left(\frac{C_{2}}{C_{1}}-\frac{C_{1}}{C_{2}}\right)+9 .
$$




\section{References}

1. Wood, D. L.; Ferguson, J.; Knox, K.; Dillon, J. F., Crystal-Field Spectra of d3,7

Ions. III. Spectrum of Cr3+ in Various Octahedral Crystal Fields, J. Chem. Phys. 1963,

$39(4), 890-898$.

2. Alexander, J. J.; Gray, H. B., Electronic structures of hexacyanometalate complexes, J. Am. Chem. Soc. 1968, 90 (16), 4260-4271.

3. Linhard, M., Über Lichtabsorption und Konstitution Anorganischer Komplexsalze, Z. Elektrochem. Angew. Phys. Chem. 1944, 50 (9-10), 224-238.

4. Schläfer, H. L., in Zeitschrift für Physikalische Chemie (1957), Vol. 11, pp. 65-77. 